\title{
Revisiting Jackson-Hunt calculations: Unified theoretical analysis for generic multi-phase growth in a multi-component system
}

\author{
Arka Lahiri $^{\mathrm{a}, *}$, Abhik Choudhury ${ }^{\mathrm{a}}$ \\ ${ }^{a}$ Department of Materials Engineering, Indian Institute of Science, Bangalore - 560 012, India.
}

\begin{abstract}
A straight-forward extension of the Jackson-Hunt theory for directionally solidifying multi-phase growth where the number of components exceeds the number of solid phases becomes difficult on account of the absence of the required number of equations to determine the boundary layer compositions ahead of the interface. In this paper, we therefore revisit the Jackson-Hunt(JH) type calculations for any given situation of multi-phase growth in a multi-component system and self-consistently derive the variations of the compositions of the solid phases as well as their volume fractions, which grow such that the composite solid-liquid interface is isothermal. This allows us to unify the (JH) calculation schemes for both in-variant as well as multi-variant eutectic reactions. The derived analytical expressions are then utilized to study the effect of dissimilar solute diffusivities and interfacial energies on the undercoolings and the solidified fractions. We also perform phase field simulations to confirm our theoretical predictions and find a good agreement between our analytical calculations and model predictions for model symmetric alloys as well as for a particular Ni-Al-Zr alloy.
\end{abstract} (1)

Keywords: Phase-field; Jackson-Hunt; multi-component; multi-phase; multi-variant; in-variant; eutectic

\section{Introduction}

Eutectic solidification in a generic multi-component alloy, where two or more solids exhibit coupled growth, can be associated with degrees of freedom greater than or equal to zero. Experimentally, invariant (zero degrees of freedom) eutectic reactions have been observed in [1, 2, 3, 4, 5, 6, 7, 8, 9, 10, 11, 12, 13, Our theoretical understanding of invariant eutectic reactions is fairly advanced for binary [14, 15, 16, 17] as well as for ternary systems [18, 19]. In these studies, the solid phase fractions are assumed to be the ones predicted by the equilibrium phase diagram at the invariant temperature. This allows the determination of the magnitude of the composition boundary layers at

\footnotetext{
* Corresponding author

Email addresses: arka@platinum.materials.iisc.ernet.in (Arka Lahiri), abhiknc@materials.iisc.ernet.in (Abhik Choudhury)
}

the eutectic front from the conditions of equality of undercoolings at different solid-liquid interfaces.

In contrast to binary alloys, multi-component alloy systems can display eutectic reactions which are not invariant (possessing degrees of freedom greater than zero). An example of such a reaction is the concurrent solidification of two phases in ternary alloys, which possesses a single degree of freedom, and is known as the monovariant (or univariant) eutectic. This reaction exists over a range of temperatures in the equilibrium phase diagram compared to its invariant counterpart in binary. Experimental studies in several multi-component systems report the existence of such multivariant reactions [20, 21, 4, 22, 23, 24, 25. Multivariant eutectics are also susceptible to MullinsSekerka [26] like destabilization of the solidification front in the presence of one or more impurity components leading to the formation of eutectic cells or colonies as seen 
in [27, 28]. Eutectic cells have been studied theoretically and numerically in 29] and [30, respectively.

The theoretical development for multi-variant eutectic reactions have mainly been centered around ternary monovariant eutectics. An attempt to extend the JH-type calculations to explain monovariant eutectics leads to an underdetermined system where the number of unknowns (for describing the magnitude of the solute boundary layers) exceed the equations (the equality of interfacial undercoolings at different solid-liquid interfaces). This marks a departure from the theories of invariant eutectics [17, 18, 19], where there are enough equations as unknowns to render the system consistent.

McCartney et al. 31] are the first to circumvent this difficulty by introducing an additional constraint relating the magnitude of the composition boundary layers of the two independent components assuming the solid phase fractions to be given by the equilibrium phase diagram. The under-determined nature of the problem of ternary monovariant eutectics urge a reduction in the number of the unknowns in the problem by expressing them as functions of the solid phase volume fractions. Thus, the solid phase fractions are no longer determined by the equilibrium phase diagram but by the growth dynamics. The dynamic selection of solid phase fractions during growth being an experimental fact valid for invariant and noninvariant eutectics alike, prompted several theoretical studies which attempt to understand eutectic solidification dynamics as functions of solid phase fractions. Donaghey and Tiller 32 calculate the composition fields in the liquid as functions of the solid phase fractions for binary as well as ternary systems, which are then utilized by Ludwig and Leibbrandt 33] to demonstrate the dependence of interfacial undercoolings on solid phase fractions, but for binary systems only. Magnin and Trivedi 34] consider different densities of the eutectic solid phases for a binary eutectic to derive the curvature at each point of the eutectic solidification front required to maintain an isothermal solid-liquid interface. These curvatures when averaged over the two eutectic solids, lead to contact angles different from what is predicted by the criterion of mechanical equilibrium at the triple points, constituting a driving force for a dynamic selection of volume fractions of solid phases different from what is predicted by the equilibrium phase diagram. For ternary monovariant eutectics, De Wilde et al. 35. treat a particular solid phase fraction as a material parameter which is then varied to obtain the corresponding variation in the growth dynamics at the extremum condition of minimum undercooling of the eutectic front.

A couple of recent theoretical studies have a gone a step further by presenting a method to compute the solid phase fractions as a key step to determine non-invariant eutectic growth involving two solid phases in a generic multicomponent alloy. The study by Catalina et al. 36] presents a linearized theory in this regard without allowing for the changes in composition in one of the solidifying phases. Here, the solid phase fractions are determined from the criterion of equal undercoolings at the two solid-liquid interfaces. A more rigorous extension is provided recently by Senninger and Voorhees [37], where they take into account the composition variations of both the solid phases. Although, our work shares a similar spirit in this aspect, we present an alternate derivation. One of the major differences is that we relate the deviations of the phase compositions to the departures of the diffusion potentials and temperature and thereby the functional dependence between the variations of the solid and liquid compositions is more elegantly retrieved. Secondly, our theory is applicable for any generic multi-phase eutectic growing with a lamellar arrangement which is in contrast to the work of Senninger and Voorhees [37, who limit themselves to twophase growth. In addition, we verify our analytical calculations with phase-field simulations considering model symmetric alloys as well as a Ni-Al-Zr alloy. In all the studies mentioned above, the effect of solute diffusivities in modifying the selection of solid phase fractions have not 
been explored. We explore this aspect using our phasefield simulations as well as analytical calculations.

\section{Analytical theory}

\subsection{The Jackson Hunt calculation}

In order to motivate our present work let us re-visit the main results of the classical Jackson-Hunt analysis as detailed in [17], for deriving the undercooling vs spacing relationships for two-phase growth in a binary alloy. The situation is modeled by considering a repeating representative unit of two phases $\alpha$ and $\beta$ growing in a directional solidification set-up where the imposed temperature gradient $(\mathrm{G})$ at the interface traverses with a velocity $\mathrm{V}$, that sets the rate of solidification. The undercooling at each interface can be written as,

$$
\Delta T^{\nu}=-m_{B}^{\nu}\left(\widetilde{c_{B}^{\nu}}-c_{B}^{E}\right)+\Gamma_{\nu} \widetilde{\kappa_{\nu}}
$$

where, $\widetilde{c_{B}^{\nu}}$ represents the average composition in the liquid in local equilibrium with the $\nu$-th phase and $c_{B}^{E}$ represents the eutectic composition. $m_{B}^{\nu}$ is the liquidus slope. $\Gamma_{\nu}$ and $\kappa_{\nu}$ denote the Gibbs-Thomson coefficient and the interfacial curvature, respectively.

We start by writing the composition profiles as a Fourier series with amplitudes that are determined from the condition that the composition profiles obey both the governing equation and the Stefan condition. A corresponding generic analysis for invariant eutectic growth in multicomponent systems is laid out in [19], where expressions for all the amplitudes apart from the zeroth order mode (representing the boundary layer) can be determined from the inverse Fourier transform. In order to understand the difficulty in determining the amplitude of the zeroth order, we first inspect the expression obtained by performing an inverse Fourier transform of the same, written as, $B_{0}=\left[c_{B}^{\alpha l} \eta_{\alpha}+c_{B}^{\beta l}\left(1-\eta_{\alpha}\right)\right]-\left[c_{B}^{\alpha} \eta_{\alpha}+c_{B}^{\beta}\left(1-\eta_{\alpha}\right)\right]$, where $c_{B}^{\alpha / \beta, l}$ represents the liquid compositions in equilibrium with the $\alpha / \beta$ interfaces, $\eta_{\alpha}$ is the volume fraction of the $\alpha$ phase and $B_{0}$ denotes the boundary layer amplitude corresponding to the component $B$. If one uses the volume fractions and compositions at the eutectic temperature (as for the other Fourier modes) for determining the boundary layer composition $B_{0}$ (far-field composition is at the eutectic) it would result in zero and the corresponding undercoolings at the interface would not be equal. This calculation, would also be physically incorrect, as the phase compositions deviate from their values at the eutectic temperature. Jackson and Hunt in their analysis, treat this difficulty by keeping the $B_{0}$ as an unknown which is fixed by the condition that the undercoolings at both solid-liquid interfaces are equal, while the volume fractions $\eta_{\alpha}$ at the eutectic temperature are utilized for computing both the constitutional and curvature undercoolings. In general, one can solve this problem of invariant growth for a multicomponent system as in [19, where it has been shown to agree well with experiments as well as phase-field simulations.

For the mono-variant reaction however, for instance in a two-phase growth in a ternary alloy, there would be two boundary layer compositions, whereas the equality of a common undercooling imparts only a single equation, thereby the system of equations become under-determined. The system of equations can only be made deterministic by invoking the functional dependence of the boundary layer compositions on the phase compositions and the solidfractions. This motivates our present derivation, which in spirit unifies the theories of in-variant and multi-variant eutectic growth.

\subsection{Theory}

The following discussion is generic to a directionally solidifying multi-component alloy of $K$ components (with $K-1$ of them being independent), displaying a eutectic reaction with $N$ solid phases, possessing a degree of freedom given by $F=K-N$. Though, we present the theory 
assuming independent diffusion of solutes in the liquid (no diffusion in the solid), it can be considered to be representative of a system with non-zero off-diagonal terms in the diffusivity matrix, when such an analysis is carried out in the basis system of the eigenvectors of the diffusivity matrix. In the following discussion, the indices $i$ and $j$ are reserved for solutes, while $\nu$ and $p$ denote the solid phases appearing due to eutectic solidification. Assuming a flat interface, the composition variation in the liquid is of the form [19],

$$
c_{i}=c_{i}^{\infty}+\sum_{n=-\infty}^{n=\infty} I_{n} e^{\hat{i} k_{n} x-q_{n}^{i} z}
$$

where, $\hat{i}=\sqrt{-1}$, and $k_{n}=2 \pi n / \lambda$, are wavenumbers characterizing the variation of solute concentrations in the liquid across a solid-liquid interface aligned along $x$ with the eutectic solids growing in $z$. Conformity of Eq. 2 to the stationary form of the diffusion equation given below,

$$
V \frac{\partial c_{i}}{\partial z}+D_{i i} \nabla^{2} c_{i}=0
$$

leads to,

$$
q_{n}^{i}=\left(\frac{V}{2 D_{i i}}\right)+\sqrt{k_{n}^{2}+\left(\frac{V}{2 D_{i i}}\right)^{2}},
$$

where $D_{i i}$ denotes the diffusivity of the $i$ th component and $V$ represents the sample pulling velocity. Following the discussion in [19], a single wavelength of the eutectic consists of $M$ units $(M>=N)$ of the eutectic solids with each one of the $M$ units corresponding to one of the $N$ phases. The periodic variation starts at $x_{0}=0$ and terminates at $x_{M}=1$ with the width of the $\nu$-th unit being given by $\left(x_{\nu}-x_{\nu-1}\right) \lambda$; the entire wavelength being $\left(x_{M}-x_{0}\right) \lambda$. Thus, the volume fraction of a particular phase $p$, denoted by $\eta_{p}$, can be calculated from a single wavelength of the eutectic lamellae as,

$$
\eta_{p}=\sum_{\nu=0}^{M-1}\left(x_{\nu+1}-x_{\nu}\right) \delta_{\nu p}
$$

where,

$$
\delta_{\nu p}= \begin{cases}1, & \text { if } \nu=p \\ 0, & \text { if } \nu \neq p\end{cases}
$$

The mass balance across a particular location at the solid-liquid interface for the $\nu$-th unit can be written as,

$$
V \Delta c_{i}^{\nu}=-\left.D_{i i} \frac{\partial c_{i}}{\partial z}\right|_{z=0, x_{\nu}^{*}}
$$

with $\Delta c_{i}^{\nu}=c_{i}^{\nu l}-c_{i}^{\nu}$, where $c_{i}^{\nu l}$ and $c_{i}^{\nu}$ denote the liquid and the solid compositions in local equilibrium at a location $x_{\nu}^{*}$ on the $\nu-l$ interface, respectively. An expression for the Fourier constants $I_{n}$ is obtained by invoking the orthogonality of the Fourier basis functions while integrating Eq. 7 over an entire period $(\lambda)$ of the eutectic, leading to,

$$
q_{n}^{i} I_{n} \lambda=\frac{2}{l_{i}} \sum_{\nu=0}^{M-1} \int_{x_{\nu} \lambda}^{x_{\nu+1} \lambda} \exp \left(-i k_{n} x\right) \Delta c_{i}^{\nu} d x
$$

where, $l_{i}=2 D_{i i} / V$, is the diffusion length associated with the $i$-th component. For the mode corresponding to $n=0$, Eq. 8 yields for the $i$-th component,

$$
I_{0}=\frac{1}{\lambda} \sum_{\nu=0}^{M-1} \int_{x_{\nu} \lambda}^{x_{\nu+1} \lambda} \Delta c_{i}^{\nu} d x
$$

It is beneficial to define average compositions in front of a particular phase $p$ as,

$$
\overline{\Delta c_{i}^{p}}=\frac{\sum_{\nu=0}^{M-1} \delta_{\nu p} \int_{x_{\nu} \lambda}^{x_{\nu+1} \lambda} \Delta c_{i}^{\nu} d x}{\sum_{\nu=0}^{M-1}\left(x_{\nu+1}-x_{\nu}\right) \lambda \delta_{\nu p}}
$$

which allows us to re-express Eq. 9 as,

$$
I_{0}=\sum_{p=1}^{N} \eta_{p} \overline{\Delta c_{i}^{p}}
$$

Similarly, the average interfacial composition in the solid $\left(\overline{c_{i}^{p}}\right)$ and the liquid $\overline{c_{i}^{p l}}$ ahead of it is defined as,

$$
\overline{c_{i}^{p, p l}}=\frac{\sum_{\nu=0}^{M-1} \delta_{\nu p} \int_{x_{\nu} \lambda}^{x_{\nu+1} \lambda} c_{i}^{\nu, \nu l} d x}{\sum_{\nu=0}^{M-1}\left(x_{\nu+1}-x_{\nu}\right) \lambda \delta_{\nu p}} .
$$

The theory in [19] following that of Jackson \& Hunt 17] provides an expression for $\overline{c_{i}^{p l}}$, which has the form,

$$
\begin{aligned}
\overline{c_{i}^{p l}} & =c_{i}^{\infty}+I_{0} \\
& +\frac{\lambda}{\eta_{p} l_{i}} f_{i}\left(P_{1}\left(\eta_{1}, \cdots, \eta_{N}\right), \cdots, P_{r}\left(\eta_{1}, \cdots, \eta_{N}\right),\right. \\
& \left.\overline{\Delta c_{i}^{1}}, \cdots, \overline{\Delta c_{i}^{N}}\right)
\end{aligned}
$$

where, each of one of the $k$ infinite series' $P_{k}\left(\eta_{p}\right), k=$ $1, \cdots, r, p=1, \cdots, N$, are composed of terms which are 
trigonometric functions of $\eta_{p}$. The value of $r$ and the form of $P_{k}\left(\eta_{p}\right)$ are determined by the number and repetitions of solid phases in a single periodic unit of wavelength $\lambda$. It must be mentioned at this point that the term $I_{0}$ represents the principal term determining the liquid compositions $\overline{c_{i}^{p l}}$ at the flat interface, with the secondary influence being due to that of the higher order modes averaged over the lamellar widths denoted by the final term in the RHS of Eq. 13. An example of such a term for a ternary monovariant eutectic [19],

$$
\begin{aligned}
f_{i} & =2 P\left(\eta_{\alpha}\right)\left(\overline{\Delta c_{i}^{\alpha}}-\overline{\Delta c_{i}^{\beta}}\right) \\
P\left(\eta_{\beta}\right) & =P\left(\eta_{\alpha}\right)=\sum_{n=1}^{\infty} \frac{1}{(\pi n)^{3}} \sin ^{2}\left(\pi n \eta_{\alpha}\right) .
\end{aligned}
$$

The average undercooling $\left(\overline{\Delta T^{p}}\right)$ ahead of a particular solid $(p)$-liquid $(l)$ interface is given by,

$$
\overline{\Delta T^{p}}=T^{*}-T^{p}=\sum_{i=0}^{K-1} m_{i}^{p}\left(c_{i}^{l, *}-\overline{c_{i}^{p l}}\right)+\Gamma_{p} \overline{\kappa_{p}},
$$

where, $m_{i}^{p}$ are the liquidus slopes, $\Gamma_{p}$ denotes the GibbsThomson coefficient with the average curvature of the solid $(p)$ liquid interface $\left(\overline{\kappa_{p}}\right)$ given by,

$$
\overline{\kappa_{p}}=\frac{2 \sin \overline{\theta_{p m}}}{\eta_{p} \lambda}
$$

where, $\overline{\theta_{p m}}$ is the angle made by the tangent to the $\operatorname{solid}(p)$ liquid $(l)$ interface and the horizontal towards the side of the $p$-th phase when located adjacent to the $m$-th phase, and averaged over all such contiguous arrangements of the solid phases $m$ and $p$ in the entire period.

The fact that the imposed thermal gradient has a length scale much larger than the lamellar width, implies the growth of all the eutectic solids at equal undercoolings, which can be expressed as,

$$
\overline{\Delta T^{1}}=\overline{\Delta T^{2}}=\cdots=\overline{\Delta T^{N}}=\overline{\Delta T} .
$$

Also, the sum of volume fractions of the phases in a single period of the lamellae must be equal to unity,

$$
\sum_{p=1}^{N} \eta_{p}=1
$$

Here, one needs to solve the Eqs. 11, 13, 16, 18 and 19 simultaneously, in order to retrieve the phase compositions, the solid fractions and the undercooling of the eutectic growth front. For this, we need to describe the functional dependence between the solid compositions $\overline{c_{i}^{p}}$ and the liquid compositions $\overline{c_{i}^{p l}}$. We do this by calling upon the relations of the phase compositions $\overline{c_{i}^{p, p l}}\left(\overline{\mu_{i}^{p}}, T\right)$, where $\overline{\mu_{i}^{p}}$ corresponds to the diffusion potential of $p-l$ equilibrium, averaged over all occurrences of the solid phase $p$ in a periodic unit of the eutectic. This then reduces the above system, Eqs. 11, 13, 16, 18 and 19 in terms of $\overline{\mu_{i}^{p}}, T$, and $\eta_{p}$

A point to note here is that, Senninger and Voorhees [37, replace the Eqs 11 with a mass conservation constraint. Mass conservation is implicit in our set of equations. This can be seen by considering only those $N$ equations out of the $N(K-1)$ in Eqs 13 which represent the composition fields of a particular component $i$ in the liquid in equilibrium with different solids $(p)$. Summing over - all such equations after multiplying both sides of each of them with the respective volume fractions $\eta_{p}$, gives $I_{0}=\sum_{p} \overline{c_{i}^{p l}} \eta_{p}-c_{i}^{\infty}$. This along with Eqs 11 implies that $\sum_{p} \overline{c_{i}^{p}} \eta_{p}=c_{i}^{\infty}$, which is the mass conservation equation used by Senninger and Voorhees [37.

However, given that the thermodynamical relations $\overline{c_{i}^{p, p l}}\left(\overline{\mu_{i}^{p}}, T\right)$ are routinely non-linear, the resultant set of equations become difficult to resolve. Given that most departures from equilibrium in case of eutectic reactions are small, we therefore linearize our set of equations about the chosen eutectic temperature $T^{*}$, the average diffusion potentials $\mu_{j}^{p, *}$ and the solid phase fractions $\eta_{p}^{*}$, corresponding to $T^{*}$. It must be noted that the equilibrium solid and liquid phase compositions corresponding to $T^{*}, \mu_{j}^{p, *}$ and $\eta_{p}^{*}$, are $c_{i}^{p, *}$ and $c_{i}^{p l, *}$, respectively. This results in a set of linear equations which can be solved for, consistently. 


\subsection{Linearized Theory}

We express the average compositions in the solid $\left(\overline{c_{i}^{p}}\right)$ and the liquid $\left(\overline{c_{i}^{p l}}\right)$ as functions of diffusion potentials $\left(\overline{\mu_{j}^{p}}\right)$ as,

$$
\overline{c_{i}^{p}}=c_{i}^{p, *}+\sum_{j=1}^{K-1}\left[\frac{\partial c_{i}^{p}}{\partial \mu_{j}}\right]_{\mu_{j}^{p, *}} \overline{\Delta \mu_{j}^{p}}-\left.\frac{\partial c_{i}^{p}}{\partial T}\right|_{T^{*}} \overline{\Delta T^{p}},
$$

and,

$$
\overline{c_{i}^{p l}}=c_{i}^{p l, *}+\sum_{j=1}^{K-1}\left[\frac{\partial c_{i}^{l}}{\partial \mu_{j}}\right]_{\mu_{j}^{p, *}} \overline{\Delta \mu_{j}^{p}}-\left.\frac{\partial c_{i}^{l}}{\partial T}\right|_{T^{*}} \overline{\Delta T^{p}},
$$

where, $\overline{\Delta \mu_{j}^{p}}=\overline{\mu_{j}^{p}}-\mu_{j}^{p \text {,* }}$, denote the change in average diffusion potentials from their values at the chosen eutectic point $\mu_{j}^{p, *}$, under an additional constraint of constant $\left[\partial c_{i} / \partial \mu_{j}\right]$ matrices. The vector $\partial c_{i}^{p} / \partial T$ determine the change in composition with temperature at constant diffusion potentials.

Employing Einstein's indicial notation which conveys summation over repeated indices (except for $p$ in our analysis, which denotes a particular phase), the above equations can be written as,

$$
\overline{c_{i}^{p}}=c_{i}^{p, *}+\chi_{i j}^{p} \overline{\Delta \mu_{j}^{p}}-\zeta_{i}^{p} \overline{\Delta T^{p}}
$$

and,

$$
\overline{c_{i}^{p l}}=c_{i}^{p l, *}+\chi_{i j}^{l} \overline{\Delta \mu_{j}^{p}}-\zeta_{i}^{l} \overline{\Delta T^{p}}
$$

where,

$$
\begin{gathered}
\chi_{i j}^{p}=\left.\frac{\partial c_{i}^{p}}{\partial \mu_{j}}\right|_{\mu_{j}^{p, *}}, \\
\chi_{i j}^{l}=\left.\frac{\partial c_{i}^{l}}{\partial \mu_{j}}\right|_{\mu_{j}^{p, *}}, \\
\zeta_{i}^{p}=\left.\frac{\partial c_{i}^{p}}{\partial T}\right|_{T^{*}}, \\
\zeta_{i}^{l}=\left.\frac{\partial c_{i}^{l}}{\partial T}\right|_{T^{*}} .
\end{gathered}
$$

Thus, the difference in the average compositions of the solid and the liquid as obtained from Eqs. 20, 21, 22, and 23 .

$$
\begin{aligned}
\overline{\Delta c_{i}^{p}} & =\overline{c_{i}^{p l}}-\overline{c_{i}^{p}} \\
& =\left(c_{i}^{p l, *}-c_{i}^{p, *}\right)+\sum_{j=1}^{K-1}\left(\frac{\partial c_{i}^{l}}{\partial \mu_{j}}-\frac{\partial c_{i}^{p}}{\partial \mu_{j}}\right)_{\mu_{j}^{p, *}} \overline{\Delta \mu_{j}^{p}} \\
& -\left(\frac{\partial c_{i}^{l}}{\partial T}-\frac{\partial c_{i}^{p}}{\partial T}\right)_{T^{*}} \overline{\Delta T^{p}} \\
& =\Delta c_{i}^{p, *}+\Delta \chi_{i j}^{p} \overline{\Delta \mu_{j}^{p}}-\Delta \zeta_{i}^{p} \overline{\Delta T^{p}}
\end{aligned}
$$

where, to obtain the last equality expressed in indicial notation, we have used,

$$
\begin{aligned}
\Delta c_{i}^{p, *} & =c_{i}^{p l, *}-c_{i}^{p, *}, \\
\Delta \chi_{i j}^{p} & =\chi_{i j}^{l}-\chi_{i j}^{p}, \\
\Delta \zeta_{i}^{p} & =\zeta_{i}^{l}-\zeta_{i}^{p} .
\end{aligned}
$$

Using, Eqs. 22, 23 and 28, the $2 N(K-1)$ composition variables have been expressed as functions of $N(K-1)$ intensive variables in the form of change in diffusion potentials $\overline{\Delta \mu_{j}^{p}}$, which can be further related to $\Delta \eta_{p}\left(=\eta_{p}-\eta_{p}^{*}\right)$ and $\overline{\Delta T^{p}}$ by invoking equality of Eqs. 13 and 21 which provide additional $N(K-1)$ equations, stated in the indicial notation (with no sum over $p$ and $i$ ) as,

$$
\begin{aligned}
\overline{c_{i}^{p l}} & =c_{i}^{p l, *}+\chi_{i j}^{l} \overline{\Delta \mu_{j}^{p}}-\zeta_{i}^{l} \overline{\Delta T^{p}} \\
& =c_{i}^{\infty}+I_{0} \\
& +\frac{\lambda}{\eta_{p} l_{i}} f_{i}\left(P_{1}\left(\eta_{1}, \cdots, \eta_{N}\right), \cdots, P_{r}\left(\eta_{1}, \cdots, \eta_{N}\right),\right. \\
& \left.\overline{\Delta c_{i}^{1}}, \cdots, \overline{\Delta c_{i}^{N}}\right)
\end{aligned}
$$

The RHS of Eq. 32 (or Eq. 13), is in general non-linear in $\overline{\Delta \mu_{j}^{p}}, \Delta \eta_{p}$ and $\overline{\Delta T^{p}}$. Thus, to express $\overline{\Delta \mu_{j}^{p}}$ as an explicit function of $\Delta \eta_{p}$ and $\overline{\Delta T^{p}}$, we linearly expand each term in the RHS of Eq. 32 starting with $I_{0}$, given by,

$$
\begin{aligned}
I_{0} & =I_{0}^{*}+\left.\sum_{m=1}^{N} \frac{\partial I_{0}}{\partial \overline{\Delta c_{i}^{m}}} \sum_{j=1}^{K-1} \frac{\partial \overline{\Delta c_{i}^{m}}}{\partial \mu_{j}}\right|_{\mu_{j}^{m, *}} \overline{\Delta \mu_{j}^{m}} \\
& -\left.\sum_{m=1}^{N} \frac{\partial I_{0}}{\partial \overline{\Delta c_{i}^{m}}} \frac{\partial \overline{\Delta c_{i}^{m}}}{\partial T}\right|_{T^{*}} \overline{\Delta T^{m}} \\
& +\left.\sum_{m=1}^{N} \frac{\partial I_{0}}{\partial \eta_{m}}\right|_{\eta_{m}^{*}} \Delta \eta_{m},
\end{aligned}
$$


where, $\mu_{i}^{p, *}, T^{*}$ and $\eta_{p}^{*}$ are the quantities corresponding to the equilibrium in the phase diagram. The different terms in the RHS of Eq. 33 can be computed from Eqs. 11, 28 and 31 as,

$$
\begin{aligned}
\frac{\partial I_{0}}{\partial \overline{\Delta c_{i}^{p}}} & =\eta_{p}^{*}, \\
\left.\frac{\partial \overline{\Delta c_{i}^{p}}}{\partial \mu_{j}}\right|_{\mu_{j}^{p, *}} & =\left(\frac{\partial c_{i}^{l}}{\partial \mu_{j}}-\frac{\partial c_{i}^{p}}{\partial \mu_{j}}\right)_{\mu_{j}^{p, *}}=\Delta \chi_{i j}^{p}, \\
\left.\frac{\partial \overline{\Delta c_{i}^{p}}}{\partial T}\right|_{T^{*}} & =\left(\frac{\partial c_{i}^{l}}{\partial T}-\frac{\partial c_{i}^{p}}{\partial T}\right)_{T^{*}}=\Delta \zeta_{i}^{p}, \\
\left.\frac{\partial I_{0}}{\partial \eta_{p}}\right|_{\eta_{p}^{*}} & =\Delta c_{i}^{p, *} .
\end{aligned}
$$

At this point we introduce the following quantities (no sum over $p)$,

$$
\begin{aligned}
\overline{\Delta \chi_{i j}^{p}} & =\eta_{p}^{*} \Delta \chi_{i j}^{p}, \\
\overline{\Delta \zeta_{i}^{p}} & =\eta_{p}^{*} \Delta \zeta_{i}^{p},
\end{aligned}
$$

to express Eq. 33 in indicial notation as,

$$
I_{0}=I_{0}^{*}+\overline{\Delta \chi_{i j}^{m} \Delta \mu_{j}^{m}}-\overline{\Delta \zeta_{i}^{m} \Delta T^{m}}+\Delta c_{i}^{m, *} \Delta \eta_{m}
$$

with $m$ and $j$ being the indices representing phases and components respectively, which are summed over. The second term in the RHS of Eq. 32 , being only a second-order correction to the interfacial liquid composition is assumed to be a function of $1 / \eta_{p}$ only, with all the other quantities evaluated at the conditions prevailing at the eutectic. This simplifying assumption is necessary to maintain tractability of the equations. Thus, writing,

$$
\begin{aligned}
& f_{i}=f_{i}^{*}\left(P_{1}\left(\eta_{1}^{*}, \cdots, \eta_{N}^{*}\right), \cdots, P_{r}\left(\eta_{1}^{*}, \cdots, \eta_{N}^{*}\right),\right. \\
& \left.\overline{\Delta c_{i}^{1, *}}, \cdots, \overline{\Delta c_{i}^{N, *}}\right)
\end{aligned}
$$

we re-write the linearized version of Eq. 32 indicially, as,

$$
\begin{aligned}
& \overline{c_{i}^{p l}}=c_{i}^{p l, *}+\chi_{i j}^{l} \overline{\Delta \mu_{j}^{p}}-\zeta_{i}^{l} \overline{\Delta T^{p}}= \\
& c_{i}^{\infty}+\left[I_{0}^{*}+\overline{\Delta \chi_{i j}^{m} \Delta \mu_{j}^{m}}-\overline{\Delta \zeta_{i}^{m} \Delta T^{m}}\right. \\
& \left.+\Delta c_{i}^{m, *} \Delta \eta_{m}\right]+\left[\left(\frac{\lambda}{\eta_{p}^{*}}-\frac{\lambda}{\eta_{p}^{* 2}} \Delta \eta_{p}\right) \tilde{f}_{i}\right],
\end{aligned}
$$

where,

$$
\tilde{f}_{i}=\frac{f_{i}^{*}}{l_{i}}
$$

and, $p$ is the index which represents a particular solid phase and is not summed over in Eq. 41 as well as in the following equations. The quantities enclosed in square brackets in the RHS of Eq. 41 represent terms obtained by linearizing the individual terms in the RHS of Eq. 32 . Eq. 41 can be re-written to express the diffusion potentials as a function of the interfacial undercoolings and solid phase volume fractions as,

$$
\begin{aligned}
& {\left[\chi_{i j}^{l}-\overline{\Delta \chi_{i j}^{p}}\right] \overline{\Delta \mu_{j}^{p}}-\overline{\Delta \chi_{i j}^{m^{\prime}} \Delta \mu_{j}^{m^{\prime}}}=} \\
& \left(c_{i}^{\infty}+I_{0}^{*}+\frac{\lambda}{\eta_{p}^{*}} \tilde{f}_{i}-c_{i}^{p l, *}\right) \\
& -\overline{\Delta \zeta_{i}^{m^{\prime}}} \overline{\Delta T^{m^{\prime}}}-\left(\overline{\Delta \zeta_{i}^{p}}-\zeta_{i}^{l}\right) \overline{\Delta T^{p}} \\
& +\Delta c_{i}^{m^{\prime}, *} \Delta \eta_{m^{\prime}}+\left(\Delta c_{i}^{p, *}-\frac{\lambda}{\eta_{p}^{* 2}} \tilde{f}_{i}\right) \Delta \eta_{p},
\end{aligned}
$$

where, a summation over the index $m^{\prime}$ runs from $1, \cdots, N$ leaving out $p$. So, Eq. 43 represents a system of $N(K-$ 1) equations which relates the $N(K-1) \overline{\Delta \mu_{i}^{p}}$ 's to $\Delta \eta_{p}$ 's and $\overline{\Delta T^{p}}$ 's. To describe this dependence, we utilize the linearity of Eq. 43 to write an explicit relation of the form,

$$
\overline{\Delta \mu_{i}^{p}}=\overline{R_{i}^{p}}+R_{i}^{p, T^{m}} \overline{\Delta T^{m}}+R_{i}^{p, \eta_{m}} \Delta \eta_{m}
$$

where, $\overline{R_{i}^{p}}, R_{i}^{p, T^{m}}$, and $R_{i}^{p, \eta_{m}}$ are coefficients determined by solving Eq. 43 with $m$ being the lone index which is summed over in Eq. 44. These relationships will enable the elimination of $\overline{\Delta \mu_{i}^{p l}}$ completely from the expressions of the undercoolings ahead of every solid phase given in Eq. 16. Substituting Eq. 44 into Eq. 39 we write in the indicial notation,

$$
\begin{aligned}
I_{0} & =I_{0}^{*}+\overline{\Delta \chi_{i j}^{m}}\left(\overline{R_{j}^{m}}+R_{j}^{m, T^{v}} \overline{\Delta T^{v}}+R_{j}^{m, \eta_{v}} \Delta \eta_{v}\right) \\
& -\overline{\Delta \zeta_{i}^{m} \Delta T^{m}}+\Delta c_{i}^{m, *} \Delta \eta_{m}, \\
& =\left(I_{0}^{*}+\overline{\Delta \chi_{i j}^{m} R_{j}^{m}}\right)+\left(\overline{\Delta \chi_{i j}^{m}} R_{j}^{m, T^{v}} \overline{\Delta T^{v}}-\overline{\Delta \zeta_{i}^{m} \Delta T^{m}}\right) \\
& +\left(\overline{\Delta \chi_{i j}^{m}} R_{j}^{m, \eta_{v}} \Delta \eta_{v}+\Delta c_{i}^{m, *} \Delta \eta_{m}\right)
\end{aligned}
$$


where $v$ is an index running over the phases $1, \cdots, N$ and is summed over along with the other phase index $m$. The index $j$ denoting the components is also summed over while $i$ continues to represent a particular component. Terms of similar character are collected within the parentheses in Eq. 45 .

The resulting set of equations in 16 ( $N$ in number) are then solved for the $2 N$ unknowns in $\overline{\Delta T^{p}}$ and $\Delta \eta_{p}$ under the constraints of equality of undercoolings ahead of the solid phases given by Eq. 18 ( $N-1$ in number $)$ and the sum of the volume fractions of the solid phases adding up to unity in Eq. 19 Substituting Eq. 13 into Eq. 16, we obtain, in Einstein's notation, without summing over $p$, but summming over $i$,

$$
\begin{aligned}
\overline{\Delta T^{p}} & =m_{i}^{p}\left(c_{i}^{l, *}-\overline{c_{i}^{p l}}\right)+\Gamma_{p} \overline{\kappa_{p}} \\
& =m_{i}^{p}\left[c_{i}^{l, *}-\left(c_{i}^{\infty}+I_{0}+\frac{\lambda}{\eta_{p}} \tilde{f}_{i}\right)\right] \\
& +\Gamma_{p} \overline{\kappa_{p}} .
\end{aligned}
$$

Linearizing the RHS of the above equation about equilibrium quantities and employing Eq. 45 leads to,

$$
\begin{aligned}
\overline{\Delta T^{p}} & =\left[m_{i}^{p}\left(c_{i}^{l, *}-\left(c_{i}^{\infty}+I_{0}^{*}+\overline{\Delta \chi_{i j}^{m} R_{j}^{m}}+\frac{\lambda}{\eta_{p}^{*}} \tilde{f}_{i}\right)\right)\right. \\
& \left.+\Gamma_{p} \overline{\kappa_{p}^{*}}\right] \\
& -\left[m_{i}^{p}\left(\overline{\Delta \chi_{i j}^{m}} R_{j}^{m, T^{v}} \overline{\Delta T^{v}}-\overline{\Delta \zeta_{i}^{m} \Delta T^{m}}\right)\right] \\
& -\left[m _ { i } ^ { p } \left(\overline{\Delta \chi_{i j}^{m}} R_{j}^{m, \eta_{v}} \Delta \eta_{v}+\Delta c_{i}^{m, *} \Delta \eta_{m}\right.\right. \\
& \left.\left.-\frac{\lambda}{\eta_{p}^{* 2}} \tilde{f}_{i} \Delta \eta_{p}\right)+\frac{\Gamma_{p} \overline{\kappa_{p}^{*}}}{\eta_{p}^{*}} \Delta \eta_{p}\right],
\end{aligned}
$$

where $\kappa_{p}^{*}$ is obtained by evaluating Eq. 17 for $\eta_{p}^{*}$. The three terms each enclosed in square brackets in the RHS of the above equation contain the constants, and the terms linear in $\overline{\Delta T^{m}}$ and $\Delta \eta_{m}$ respectively. We now impose Eq. 18 on
Eq. 47 to re-express it in terms of $\overline{\Delta T}$ and $\overline{\Delta \eta_{p}}$, s, as follows,

$$
\begin{aligned}
& {\left[1+m_{i}^{p}\left(\overline{\Delta \chi_{i j}^{m}} \sum_{v=1}^{N} R_{j}^{m, T^{v}}-\sum_{m=1}^{N} \overline{\Delta \zeta_{i}^{m}}\right)\right] \overline{\Delta T}} \\
& +\left[m_{i}^{p}\left(\overline{\Delta \chi_{i j}^{m}} R_{j}^{m, \eta_{p}}+\Delta c_{i}^{p, *}-\frac{\lambda}{\eta_{p}^{* 2}} \tilde{f}_{i}\right)\right. \\
& \left.+\frac{\Gamma_{p} \overline{\kappa_{p}^{*}}}{\eta_{p}^{*}}\right] \Delta \eta_{p} \\
& +\left[m_{i}^{p}\left(\overline{\Delta \chi_{i j}^{m}} R_{j}^{m, \eta_{v^{\prime}}}+\Delta c_{i}^{v^{\prime}, *}\right)\right] \Delta \eta_{v^{\prime}}= \\
& {\left[m_{i}^{p}\left(c_{i}^{l, *}-\left(c_{i}^{\infty}+I_{0}^{*}+\overline{\Delta \chi_{i j}^{m} R_{j}^{m}}+\frac{\lambda}{\eta_{p}^{*}} \tilde{f}_{i}\right)\right)\right.} \\
& \left.+\Gamma_{p} \overline{\kappa_{p}^{*}}\right]
\end{aligned}
$$

where, $v^{\prime}$ is another phase index running from 1 to $N$ except $p$ and is summed over along with the other phase index $m$. The component indices $i$ and $j$ are also summed over in Eq.48, At this stage, we will invoke Eq. 19 to eliminate $\Delta \eta_{N}$ from the above set of equations. Now, Eq. 48 represents a system of $N$ linear equations containing the same number of unknowns in $\overline{\Delta T}$ and $\Delta \eta_{1}, \cdots, \Delta \eta_{N-1}$. Solving Eq. 48 to compute $\overline{\Delta T}$ and $\Delta \eta_{1}, \cdots, \Delta \eta_{N-1}$, enables the calculation of $\overline{\Delta \mu_{j}^{1}}, \cdots, \overline{\Delta \mu_{j}^{N}}$ from Eq. 44 . Thereafter, the phase compositions can be directly obtained from Eqs. 20 and 21.

To summarize, our analytical method involves the following steps:

- For every solid-liquid equilibrium in a single period of the eutectic, the solid and the liquid compositions averaged over their corresponding lamellar widths are expressed as linear functions of the corresponding changes in diffusion potentials and undercoolings.

- We also determine the average composition in the liquid in equilibrium with the different solid phases from the Jackson-Hunt type analysis involving the superposition of multiple Fourier modes, which is again linearized about the chosen eutectic point to obtain the liquid compositions as functions of changes in diffusion potentials, undercoolings and changes in 
solid phase volume fractions. This also involves expressing the boundary layer compositions in terms of the departure of diffusion potentials and phase fractions from their corresponding values at the eutectic along with the undercooling at the solid-liquid interface.

- Using the equality of the liquid compositions obtained from the earlier steps we derive expressions of the diffusion potentials as functions of undercoolings and changes in solid phase fractions.

- Substituting for the liquid compositions using the linearized version of the Fourier series representation in terms of undercoolings, diffusion potentials and solid phase fractions into the expressions of the undercoolings at each solid-liquid interface, and enforcing the isothermal nature of the interface we compute the magnitude of the interfacial undercooling and solid phase fractions.

- Phase compositions get automatically determined due to their explicit and implicit (due to diffusion potentials) dependence on undercooling alongside their dependence on solid phase fractions.

A point to note over here is while Eq. 44 relates the deviations of the diffusion potentials $\overline{\Delta \mu_{i}^{p}}$ with the deviations of the solid fractions and the undercoolings, one can additionally invoke the condition of local thermodynamic equilibrium at the interface (including curvature) and thereby eliminate the undercoolings from the relation in Eq. 44. A similar approach has been used by Senninger and Voorhees [37]. We have tried this out as well and the results from both approaches are comparable. This completes our theoretical derivation of generic multi-component multi-phase eutectic growth. In the following section, we validate our theory against phase-field simulations of invariant and mono-variant eutectic growth.

\section{Phase field model}

Following [38, the grand potential functional $(\Omega)$ can be expressed as,

$$
\begin{aligned}
\Omega(\boldsymbol{\mu}, T, \boldsymbol{\phi}) & =\int_{V}[\Psi(\boldsymbol{\mu}, T, \boldsymbol{\phi}) \\
& \left.+\left(\epsilon a(\boldsymbol{\phi}, \nabla \boldsymbol{\phi})+\frac{1}{\epsilon} w(\boldsymbol{\phi})\right)\right] d V,
\end{aligned}
$$

where $\phi=\left[\phi_{1}, \phi_{2}, \cdots, \phi_{N}\right]$ are the phase-fields representing the spatial arrangement of $N$ phases and $\boldsymbol{\mu}=\left[\mu_{1}, \mu_{2}, \cdots, \mu_{K-1}\right]$ are the diffusion potentials associated with each one of the $K-1$ independent solutes. The functionals $w$ and $a$ represent the surface potential and the gradient energy density respectively. The minimization of $\Omega$ leads to the evolution of the spatial arrangement of the phases $(\phi)$ denoted by,

$$
\begin{aligned}
\tau \epsilon \frac{\partial \phi_{p}}{\partial t}=\epsilon & \left(\nabla \cdot \frac{\partial a(\boldsymbol{\phi}, \nabla \boldsymbol{\phi})}{\partial \nabla \phi_{p}}-\frac{\partial a(\boldsymbol{\phi}, \nabla \boldsymbol{\phi})}{\partial \phi_{p}}\right) \\
& -\frac{1}{\epsilon} \frac{\partial w(\boldsymbol{\phi})}{\partial \phi_{p}}-\frac{\partial \Psi(\boldsymbol{\mu}, T, \boldsymbol{\phi})}{\partial \phi_{p}}-\Lambda,
\end{aligned}
$$

where $\Lambda$ is calculated to ensure $\sum_{m=1}^{N} \phi_{m}=1$ at every mesh point in the simulation domain. $\tau$ is the relaxation constant with its value set based on the criterion stated in 38] and 39] to obtain a diffusion controlled interface motion.

In this model,

$$
\Delta \Psi^{m p}=\Psi^{m}-\Psi^{p}
$$

represents the driving force for a transformation of phase $m$ to $p$, with the grand-potentials of the individual phases given by,

$$
\Psi^{p}=f^{p}\left(\mathbf{c}^{p}(\boldsymbol{\mu}, T), T\right)-\sum_{i=1}^{K-1} \mu_{i} c_{i}^{p}(\boldsymbol{\mu}, T) .
$$

All the grand-potentials of the participating phases $\left(\Psi^{p}\right)$ 's at any particular point in the simulation domain are interpolated to obtain $\Psi$ as,

$$
\Psi(\boldsymbol{\mu}, T, \phi)=\sum^{p} \Psi^{p}(T, \boldsymbol{\mu}) h_{p}(\boldsymbol{\phi}),
$$


where,

$$
h_{p}(\phi)=\phi_{p}^{2}\left(3-2 \phi_{p}\right)+2 \phi_{p} \sum_{\substack{m=1, n=1, m<n, m \neq p, n \neq p}}^{N, N} \phi_{m} \phi_{n} .
$$

The gradient energy density $(a(\phi, \nabla \phi))$ in the absence of interfacial energy anisotropy can be written as,

$$
a(\boldsymbol{\phi}, \nabla \boldsymbol{\phi})=\sum_{m=1, p=1, m<p}^{N, N} \gamma_{m p}\left|q_{m p}\right|^{2},
$$

where, $\gamma_{m p}$ is the $m-p$ interfacial energy, and $q_{m p}$ is the normal vector to the $m-p$ interface, written as,

$$
q_{m p}=\phi_{m} \nabla \phi_{p}-\phi_{p} \nabla \phi_{m}
$$

The surface potential $w(\phi)$ is given by,

$$
w(\phi)= \begin{cases}\frac{16}{\pi^{2}} \sum_{\substack{m, p=1 \\(m<p) \\ N, N, N}}^{N, N} \gamma_{m p} \phi_{m} \phi_{p}+ & \\ \sum_{\substack{m, p, n=1 \\(m<p<n)}}^{N<p} \gamma_{m p} \phi_{m} \phi_{p}, & \text { if } \phi \in \sum \\ \infty, & \text { elsewhere }\end{cases}
$$

where $\sum=\left\{\boldsymbol{\phi} \mid \sum_{m=1}^{N} \phi_{m}=1\right.$ and $\left.\phi_{m} \geq 0\right\}, \gamma_{m p}$ is the surface entropy density and $\gamma_{m p n}$ is a term added to maintain the solution at an $m p$ interface strictly along the two phase interface.

The evolution of $\boldsymbol{\mu}$ is expressed as,

$$
\begin{aligned}
& \left\{\frac{\partial \mu_{i}}{\partial t}\right\}= \\
& {\left[\sum_{p} h_{p}(\phi) \frac{\partial c_{i}^{p}(\boldsymbol{\mu}, T)}{\partial \mu_{j}}\right]_{i j}^{-1}} \\
& \left\{\nabla \cdot\left(\sum_{j=1}^{K-1} M_{i j}(\phi) \nabla \mu_{j}-\mathbf{J}_{a t, i}\right)\right. \\
& \left.-\sum_{\nu} c_{i}^{p}(\boldsymbol{\mu}, T) \frac{\partial h_{p}(\boldsymbol{\phi})}{\partial t}\right\}_{j},
\end{aligned}
$$

where $i$ and $j$ iterate over the $(K-1)$ independent components. [ $\cdot]$ denotes a matrix of dimension $((K-1) \times(K-$ 1)) while $\{\cdot\}$ represents a vector of dimension $(K-1)$. The anti-trapping current $\mathbf{J}_{\mathbf{a t}, \mathbf{i}}$ has a sense and magnitude which nullifies solute trapping at the solid-liquid interface and is determined by the expressions given in [39].

The atomic mobility, $M_{i j}(\phi)$ is obtained by interpolating the individual phase mobilities as,

$$
M_{i j}(\phi)=\sum_{\nu} M_{i j}^{p} g_{p}(\phi),
$$

where the individual phase mobilities are given by,

$$
\left[M_{i j}^{p}\right]=\left[D_{i k}^{p}\right]\left[\frac{\partial c_{k}^{p}(\boldsymbol{\mu}, T)}{\partial \mu_{j}}\right],
$$

where $D_{i j}^{p}$ are the solute inter-diffusivities in the $p$-th phase and $g_{p}(\phi)$ are interpolants given as,

$$
g_{p}(\phi)=\phi_{p}^{2}\left(3-2 \phi_{p}\right) .
$$

The composition fields are obtained as functions of $\boldsymbol{\mu}$ and $\phi$ as,

$$
\begin{array}{r}
c_{i}=\sum_{p} c_{i}^{p}(\boldsymbol{\mu}, T) h_{p}(\boldsymbol{\phi}), \\
c_{i}^{p}(\boldsymbol{\mu}, T)=-V_{m} \frac{\partial \Psi_{p}(\boldsymbol{\phi}, \boldsymbol{\mu}, T)}{\partial \mu_{i}} .
\end{array}
$$

with the molar $V_{m}$ is taken to be a constant across all the components.

\section{Results: Two-solid phases in a ternary system}

In this section we employ both the analytical and phasefield models described above to study the solidification of two solid phases in a ternary alloy and compare the predictions from these two techniques for different solute interdiffusivities in the liquid. Although, the analytical theory and phase field model are general enough to describe the solidification at off-eutectic compositions, we restrict our study to eutectic compositions only.

The solid phases in the ternary monovariant eutectic are anointed as $\alpha$ and $\beta$ with the independent components constituting the ternary alloy being $A$ and $B$. All our studies are performed at a single sample pulling velocity of $V=0.01$ under an imposed thermal gradient of $G=0.0005$, with the solute diffusivities assumed to be 
negligibly small in the solids compared to that in the liquid. We will first consider a model alloy whose solid compositions are symmetrically located with respect to that of the liquid and follow it up with a similar study of a monovariant eutectic reaction in the $\mathrm{Ni}-\mathrm{Al}-\mathrm{Zr}$ alloy system.

\subsection{Calculation of $\eta_{\alpha}^{*}$ and $\eta_{\beta}^{*}$}

A three phase equilibrium ( $\alpha, \beta$ and liquid) in a ternary system is associated with a single degree of freedom as it can exist over a range of temperatures. During directional solidification, the far-field liquid composition can be found to correspond to a particular temperature $\left(T^{*}\right)$ in the equilibrium phase diagram at which it is in equilibrium with two other solid phases. If such a liquid is assumed to solidify at this temperature, the volume fractions of the resultant $\alpha$ and $\beta$ phases are what we refer to as $\eta_{\alpha}^{*}$ and $\eta_{\beta}^{*}$, respectively. As there are two independent far-field compositions in a ternary system, we invoke two artificial phase fractions $\eta_{\alpha}^{\prime}$ and $\eta_{\beta}^{\prime} \neq\left(1-\eta_{\alpha}^{\prime}\right)$ to solve for,

$$
\begin{aligned}
& c_{A}^{\infty}=c_{A}^{\alpha} \eta_{\alpha}^{\prime}+c_{A}^{\beta} \eta_{\beta}^{\prime}, \\
& c_{B}^{\infty}=c_{B}^{\alpha} \eta_{\alpha}^{\prime}+c_{B}^{\beta} \eta_{\beta}^{\prime},
\end{aligned}
$$

consistently. In general, $\eta_{\alpha}^{\prime}+\eta_{\beta}^{\prime} \neq 1$ and we compute the normalized volume fractions,

$$
\begin{aligned}
& \eta_{\alpha}^{*}=\frac{\eta_{\alpha}^{\prime}}{\eta_{\alpha}^{\prime}+\eta_{\beta}^{\prime}}, \\
& \eta_{\beta}^{*}=\frac{\eta_{\beta}^{\prime}}{\eta_{\alpha}^{\prime}+\eta_{\beta}^{\prime}},
\end{aligned}
$$

obeying $\eta_{\alpha}^{*}+\eta_{\beta}^{*}=1$ and serving as values of the volume fractions about which linearization is performed.

\subsection{Symmetric system}

To isolate and understand the effect of differences in solute diffusivities on the eutectic growth dynamics, we select a system where the equilibrium phase compositions of solid phases are symmetric with respect to the liquid composition $\left(c_{A}^{\alpha}=0.74, c_{B}^{\alpha}=0.18, c_{A}^{\beta}=0.18, c_{B}^{\beta}=\right.$ $\left.0.74, c_{A}^{l}=0.36, c_{B}^{l}=0.36\right)$. Furthermore, we choose the liquidus slopes to be consistent with this symmetry $\left(m_{A}^{\alpha}=\right.$ $\left.0.45, m_{B}^{\alpha}=0, m_{A}^{\beta}=0, m_{B}^{\beta}=0.45\right)$. We begin with the study of a system with identical $\alpha$-liquid and $\beta$-liquid interfacial energies which serves as a reference when we attempt to understand the dynamics of systems displaying dissimilar interfacial energies of the eutectic solids with liquid.

\subsubsection{Equal $\alpha$-liquid and $\beta$-liquid interfacial energies}

The equality of $\alpha$-liquid and $\beta$-liquid interfacial energies leads to $\theta_{\alpha \beta}=\theta_{\beta \alpha}=30^{\circ}$ with the Gibbs-Thomson coefficients computed to be $\Gamma_{\alpha}=\Gamma_{\beta}=0.77$ for the particular thermodynamics employed. We present analytically calculated variations of interfacial undercoolings $(\Delta T)$, solid phase volume fractions $\left(\eta_{\alpha}\right)$ and compositions of the $\alpha$ and the $\beta$ phases with lamellar width $(\lambda)$ in Fig. 1 and compare them against predictions obtained from phase field simulations. As can be seen from Fig. 1a, the analytical and phase-field calculations are in very good agreement as far as the predictions in $\lambda_{\min }$ (the lamellar width corresponding to the minimum in $\Delta T$ ) are concerned. The close agreement between the analytical theory and the phase field simulations are also evident from the variations of $\eta_{\alpha}$ versus $\lambda$ presented in Fig. $1 \mathrm{~b}$ and the variation of the average solid phase compositions in Figs. $1 \mathrm{c}$ and $1 \mathrm{~d}$. The deviations of analytically computed values of $\Delta T$ (in Fig. 1a) from those obtained from phase field simulations can be attributed to the inherent assumptions in the Jackson-Hunt calculations, where a planar interface is used to approximate the diffusion-field ahead of the solid interfaces, which are in reality curved for a system with isotropic surface energies. This mismatch has also been shown before [40, and a concomitant comparison of the phase-field method with calculations based on the boundary-integral method have proved that the predictions of the phase-field method are more accurate in this regard.

From Fig. 1a, it can be seen that lowering either of the solute diffusivities leads to a reduction in the length scale of the eutectic $\left(\lambda_{\min }\right)$ with a consequent rise in $\Delta T$. This is 


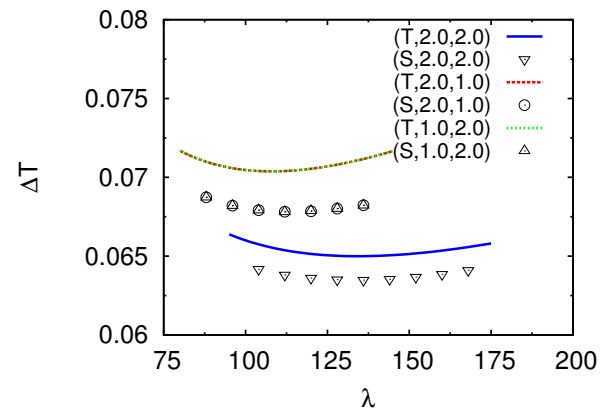

(a)

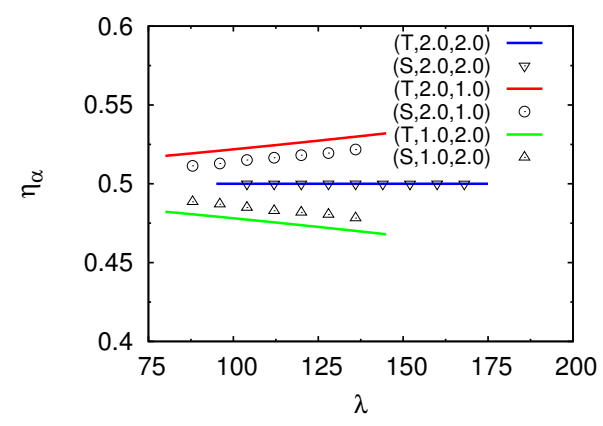

(b)

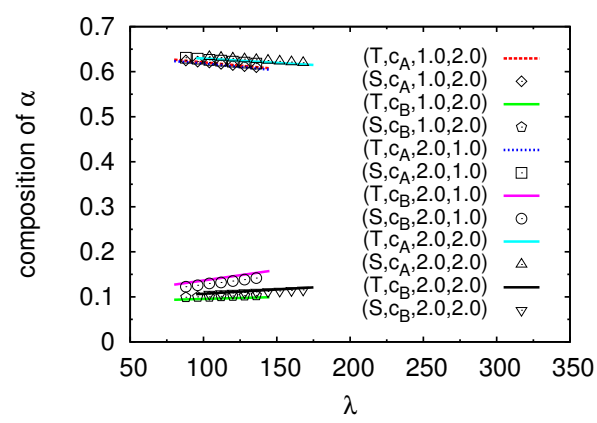

(c)

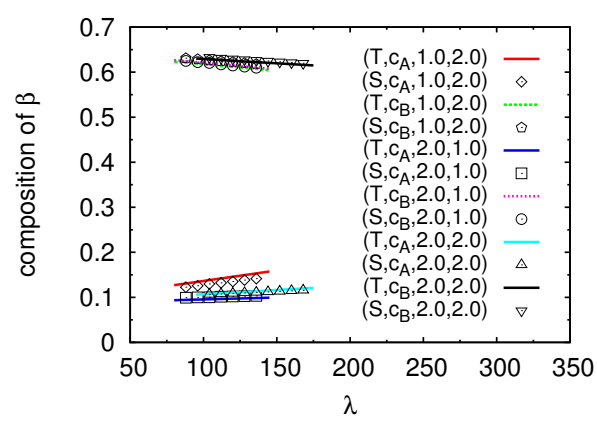

(d)

Figure 1: (Color online) Plots showing variations of (a) $\Delta T$, (b) $\eta_{\alpha}$, (c) $\alpha$ phase compositions and (d) $\beta$ phase compositions, with $\lambda$, during two phase growth in a model symmetric ternary alloy. The first position in the figure legends in (a) and (b) indicates whether the plotted data comes from theory $(\mathrm{T})$ or simulations (S); the second and third positions represent values of $D_{A A}$ and $D_{B B}$ respectively. For (c) and (d), the first position in the figure legends conveys the 12 same as in (a) and (b), the second position indicates whether the plotted variation in compositions corresponds to $A$ or $B$. The third and fourth positions in the figure legends in (c) and (d) represent the values of solutal interdiffusivities of $D_{A A}$ and $D_{B B}$ respectively. a result of a lowered effective diffusivity leading to a lower effective diffusion length. As a reflection of the inherent symmetry in the system, the theoretical calculations for $\Delta T$ vs $\lambda$ are exactly identical for $D_{A A}=2.0, D_{B B}=1.0$ and $D_{A A}=1.0, D_{B B}=2.0$.

For equal solute diffusivities the volume fractions of the eutectic solids are the same, but Fig. $1 \mathrm{~b}$ reveals that for $D_{A A}=2.0, D_{B B}=1.0, \alpha$ phase occupies a larger solid fraction $\left(\eta_{\alpha}>0.5\right)$ of the lamellar width $(\lambda)$; the same observation being valid for $\beta$ when $D_{A A}=1.0, D_{B B}=2.0$.

Physically, the change in the volume fractions can be seen as a consequence of asymmetric changes in the constitutional undercooling at each solid-liquid interface. Starting from a purely symmetric state with equal volume fractions, a situation of higher $D_{A A}$ in comparison to $D_{B B}$ would result in a lower undercooling ahead of the $\alpha-l$ interface than the $\beta-l$ interface. To recover an isothermal situation between the two interfaces, would require the $\alpha-l$ interface to assume an interfacial curvature that is greater than that acquired by the $\alpha-l$ interface when mechanical equilibrium is maintained at the trijunction. This departure from equilibrium acts as a driving force, where mechanical equilibrium is re-established through an increase in the volume fractions of the phase $\alpha$. This explains the observed variation of $\eta_{\alpha}$ with $\lambda$ in Fig. 1b. A similar argument can be made with respect to the lowering of the value of $D_{A A}$ with respect to $D_{B B}$ (see Fig. 1b), where it must be noted that as a consequence of the underlying symmetry in the system that the $\eta_{\alpha}$ vs $\lambda$ curve for $D_{A A}=1.0, D_{B B}=2.0$ can be reflected about the $\eta_{\alpha}=0.5$ line to obtain the variation for $D_{A A}=2.0, D_{B B}=1.0$. In general, any change(change in diffusivity, interfacial energies etc.) which causes an increase in the undercooling of a particular phase-liquid interface would need to be offset through an appropriate decrease in the interfacial curvature which can be affected only through a departure of the angles at the triple-point to lower values than that exists at equilibrium, keeping the same phase fractions. This 
departure acts as a driving force to establish equilibrium which is achieved by a decrease in the volume fraction of this phase.

\subsubsection{Unequal $\alpha$-liquid and $\beta$-liquid interfacial energies}

Here we probe the effect of unequal surface energies on the steady-state monovariant eutectic growth dynamics while retaining the symmetry in the phase compositions and the liquidus slopes from the system just discussed. The interfacial energies are so chosen such that $\theta_{\alpha \beta}=30^{\circ}$ and $\theta_{\beta \alpha}=45^{\circ}$ with the Gibbs-Thomson coefficients being $\Gamma_{\alpha}=0.77$ and $\Gamma_{\beta}=0.94$. Fig. 2 shows the variation in $\Delta T, \eta_{\alpha}$ and the solid phase compositions with $\lambda$ obtained from both analytical and phase field calculations.

The variation of $\Delta T$ with $\lambda$ reported in Fig. 2a presents a departure from the symmetry observed in Fig. 1a as the curves corresponding to $D_{A A}=2.0, D_{B B}=1.0$ and $D_{A A}=1.0, D_{B B}=2.0$ do not overlap. This is a consequence of the dissimilar energies of the two eutectic solidliquid interfaces. However, the variation of the phase compositions, minimum undercooling spacings and the variation of the undercoolings are similarly captured by the simulations and the theoretical calculations.

Furthermore, while the trends in the variation of the $\eta_{\alpha}$ with $\lambda$ are effectively captured for all the cases, the magnitude of variation between the simulations and theory is larger than the previous simulations with the symmetric interface properties. The reason for this is the asymmetric nature of the interface shapes, where in the phase-field simulation the $\beta-l$ interface is ahead of the $\alpha-l$ interface (see Fig 3) and thereby clearly the interfacial undercoolings of the two phases are not the same. Additionally, the departure from a planar interface is higher for the $\beta-l$ interface compared to the $\alpha-l$ interface, which also implies that this brings in added asymmetry with respect to a mismatch with the analytical calculations which are performed for a planar interface. Thereby, now any change in the interface shape which reduces the curvature of the

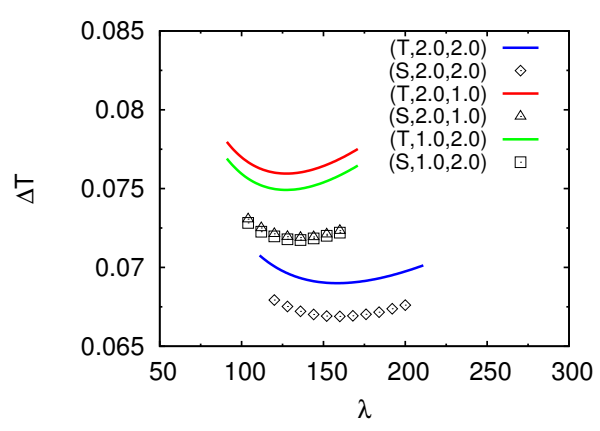

(a)

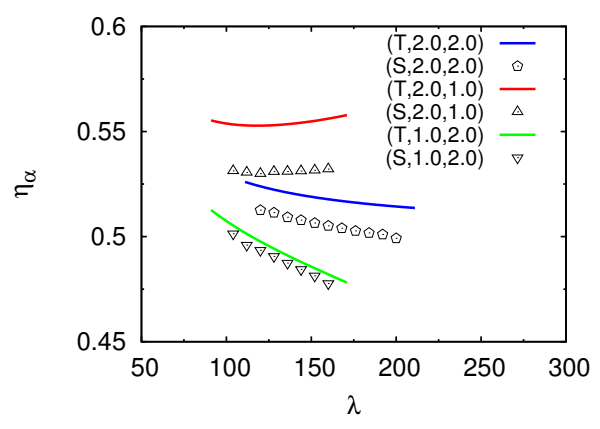

(b)

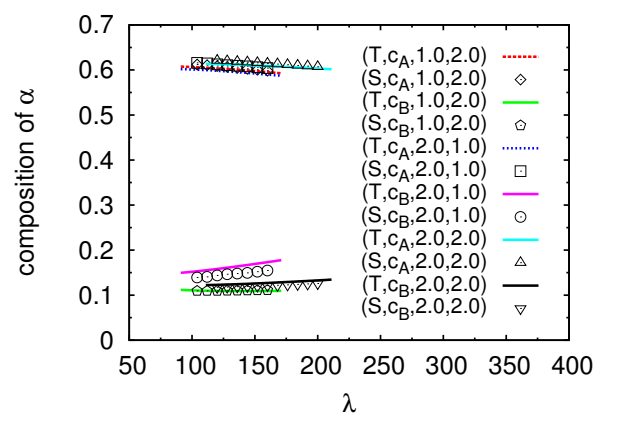

(c)

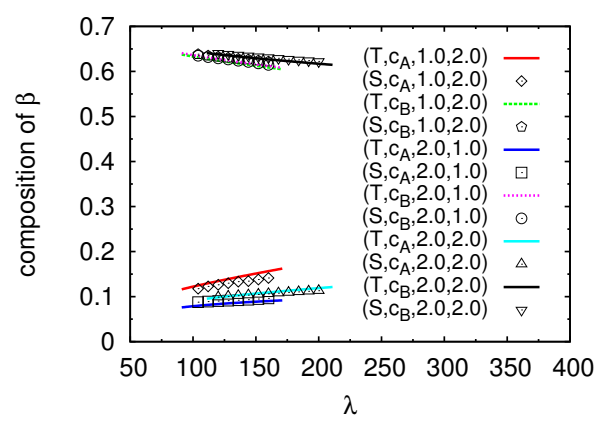

(d)

Figure 2: (Color online) Plots showing variations of (a) $\Delta T$, (b) $\eta_{\alpha}$, (c) $\alpha$ phase compositions, and (d) $\beta$ phase compositions, with $\lambda$, during two phase eutectic growth in a model symmetric ternary alloy with unequal interfacial energies. The figure legends can be interpreted in the same way as described in the caption of Fig. 1 


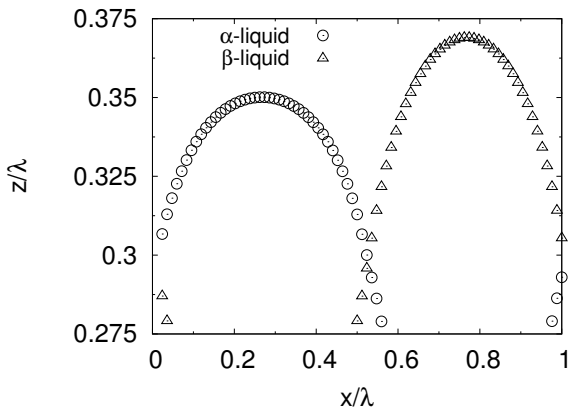

Figure 3: Plot showing the locations of the $\alpha-l$ and the $\beta-l$ interfaces.

$\beta-l$ interface decreases the disparity between the analytical calculations and phase-field simulations and additionally with increasing curvature differences between the $\alpha-l$ and $\beta-l$ interfaces, the discrepancies between the theoretical predictions and simulation results also increases. Thus, this brings to light a limitation of the analytical calculations, which work best when interfacial shapes of the solid-liquid interfaces are similar.

\subsection{Ni-Al-Zr alloy system}

In this section we study the steady-state dynamics of monovariant eutectic growth in a $\mathrm{Ni}-\mathrm{Al}-\mathrm{Zr}$ alloy at the backdrop of the insights developed in the previous section. The equilibrium phase compositions at the temperature of interest are: $c_{A}^{\alpha}=0.67, c_{B}^{\alpha}=0.32, c_{A}^{\beta}=0.74, c_{B}^{\beta}=$ $0.0034, c_{A}^{l}=0.69, c_{B}^{l}=0.19$ with the liquidus slopes being $m_{A}^{\alpha}=0.37, m_{B}^{\alpha}=1.08, m_{A}^{\beta}=-0.07, m_{B}^{\beta}=-1.0$. The Gibbs-Thomson coefficients are $\Gamma_{\alpha}=1.13, \Gamma_{\beta}=0.81$ with the contact angles $\theta_{\alpha \beta}=\theta_{\beta \alpha}=30^{\circ}$. We present the variations in $\Delta T, \eta_{\alpha}$ and the solid phase compositions in Fig. 4 .

From all the diffusivity combinations studied it can be said that the equilibrium phase compositions and the liquidus slopes are such that a higher volume fraction of $\alpha$ is the preferred morphology. Furthermore, it can be seen from Figs. 4a and 4b, that the dynamics is much more sensitive to a change in $D_{B B}$ compared to a change in $D_{A A}$. The undercoolings are found to be much higher for

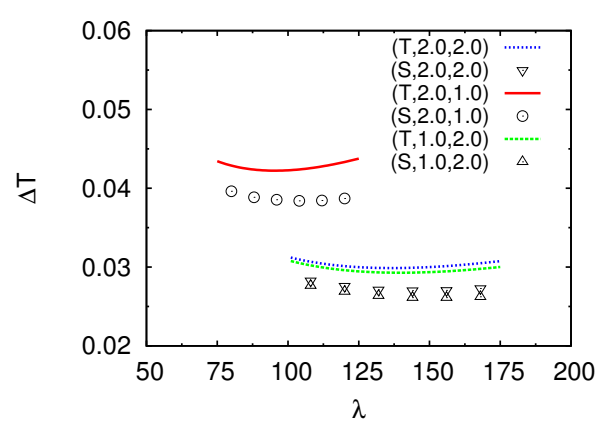

(a)

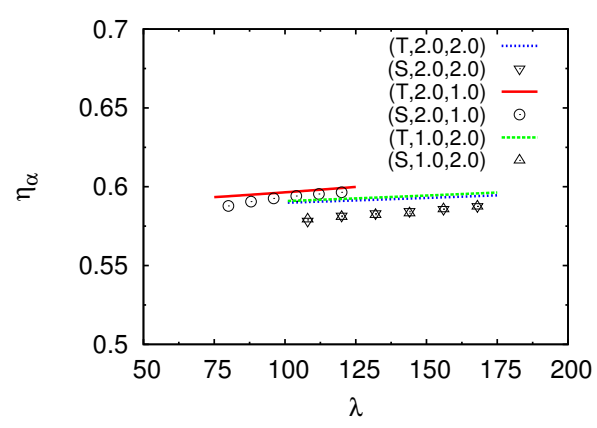

(b)

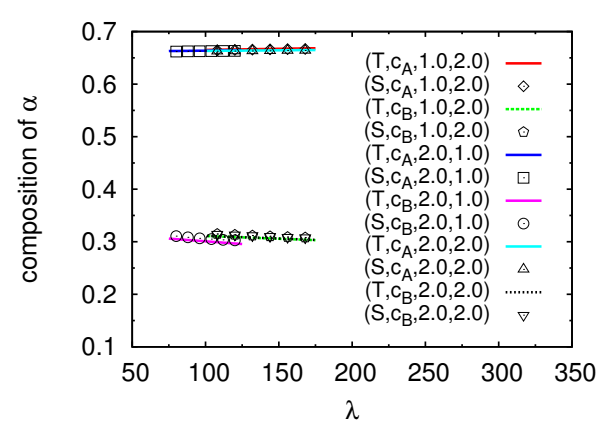

(c)

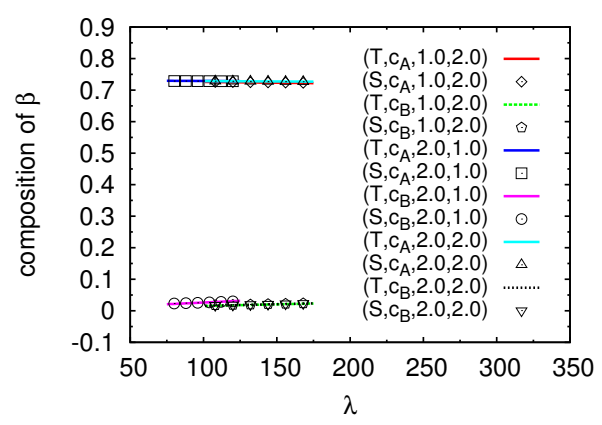

(d)

Figure 4: (Color online) Plots showing variations of (a) $\Delta T$, (b) $\eta_{\alpha}$, (c) $\alpha$ phase compositions, and (d) $\beta$ phase compositions, with $\lambda$, during two-phase growth in a Ni-Al-Zr alloy. The figure legends can be interpreted in the same way as described in the caption of Fig. 1 
$D_{A A}=2.0, D_{B B}=1.0$ than for the other two situations studied, with an accompanied shift in length scales $\left(\lambda_{\min }\right)$ towards smaller values. Also, $\eta_{\alpha}$ displays a steady rise with $\lambda$ which is much steeper for $D_{A A}=2.0, D_{B B}=1.0$ compared to the other two diffusivity configurations studied. The variations of the solid phase compositions are depicted in Fig. 4c and 4d.

\section{Results: Three phases in a ternary system}

Following up from the previous studies on two-phase mono variant growth, in this section we investigate threephase invariant growth. Contrary to two-phase growth where there is a single possibility for the lamellar arrangement of the phases, for the case of three-phase growth there exist infinitely many configurations (e.g., $\alpha \beta \gamma, \alpha \beta \alpha \gamma \ldots$..). In the following discussion, we consider two such possibilities for study, using both analytical calculations and phase-field simulations. Here we conduct simulations for the different choices of the diffusivity matrices and compare the predictions of the phase compositions and the volume fractions between the phase-field simulations and the theoretical predictions. The equilibrium phase compositions at the temperature of the invariant eutectic are: $c_{A}^{\alpha}=0.706, c_{B}^{\alpha}=0.146, c_{A}^{\beta}=0.146, c_{B}^{\beta}=0.706, c_{A}^{l}=$ $0.333, c_{B}^{l}=0.333$ with the liquidus slopes being $m_{A}^{\alpha}=$ $0.91, m_{B}^{\alpha}=0.0, m_{A}^{\beta}=0.0, m_{B}^{\beta}=0.91, m_{A}^{\gamma}=-0.91, m_{B}^{\gamma}=$ -0.91. The Gibbs-Thomson coefficients are $\Gamma_{\alpha}=\Gamma_{\beta}=$ $\Gamma_{\gamma}=1.558$ with the contact angles $\theta_{\alpha \beta}=\theta_{\beta \alpha}=\theta_{\beta \gamma}=$ $\theta_{\gamma \beta}=\theta_{\alpha \gamma}=\theta_{\gamma \alpha}=30^{\circ}$. The directional solidification conditions are kept the same as in the study of monovariant eutectic growth in ternary alloys.

We first consider the simplest arrangement $\alpha \beta \gamma$, where for the case of equal diagonal diffusivities, we get excellent agreement between our theory and phase-field simulation results (see Fig. 5), which is reflected not only in the variations of the undercooling with spacing, but also in the compositions of the phases (see Fig. 5) and in the volume fractions which given the symmetry of the phase-diagram and the diffusivities remain at $\left(\eta_{\alpha}, \eta_{\beta}, \eta_{\gamma}\right):(1 / 3,1 / 3,1 / 3)$.

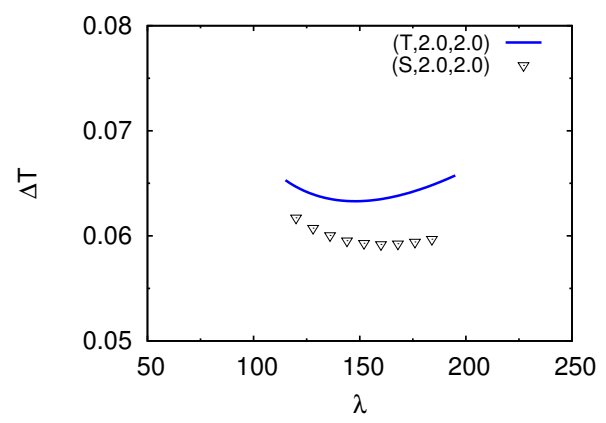

(a)

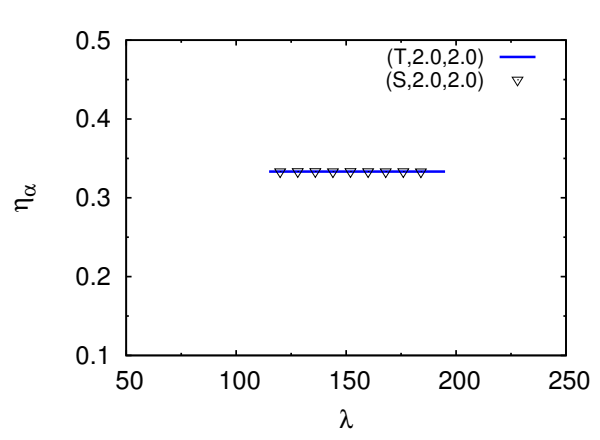

(b)

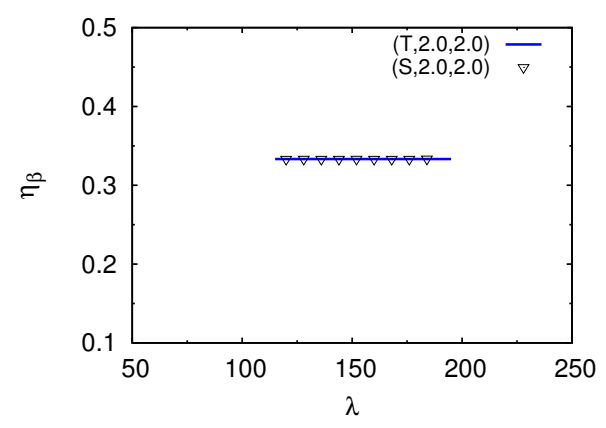

(c)

Figure 5: (Color online) Plots showing variations of (a) $\Delta T$, (b) $\eta_{\alpha}$, and (c) $\eta_{\beta}$, with $\lambda$, during three phase eutectic growth in a model symmetric ternary alloy. A single wavelength of the eutectic solids has the configuration: $\alpha \beta \gamma$. The figure legends can be interpreted in the same way as described in the caption of Fig. 1

However, for the case of unequal diffusivities, an inference from the phase-field simulations can be seen in Fig. 6 where we notice a tilt in the lamellar arrangement with respect to the growth direction.

We note that this tilt is not an "instability" that occurs beyond a spacing as has been reported during two and 


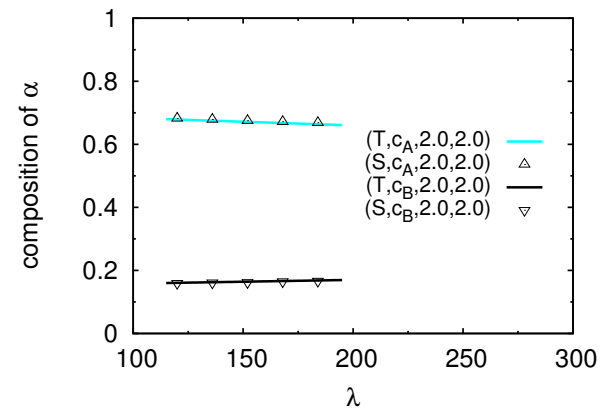

(d)

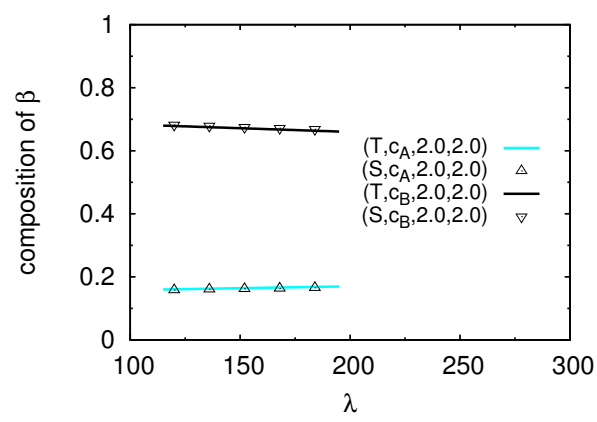

(e)

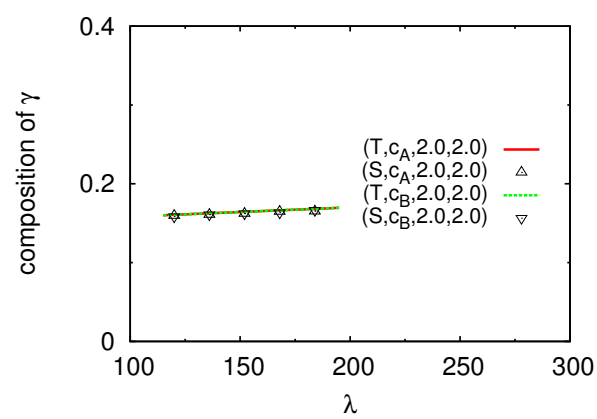

(f)

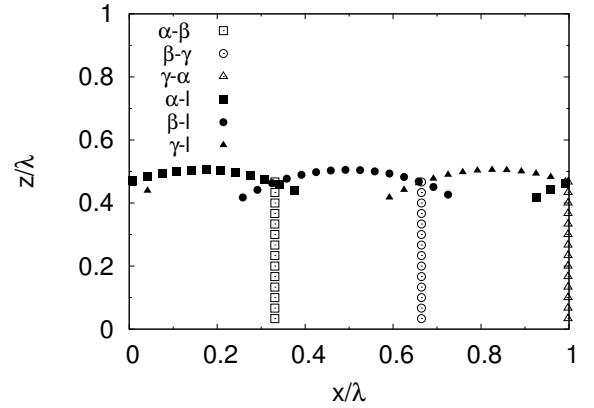

(a)

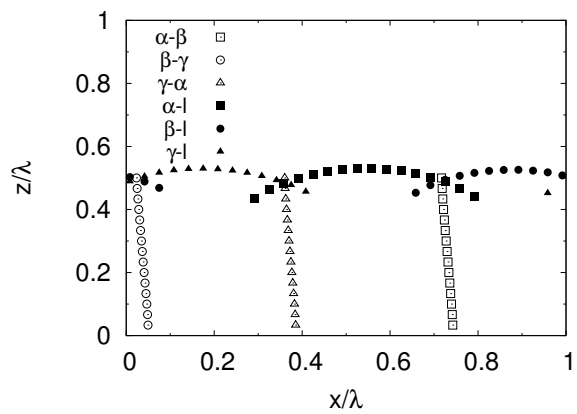

(b)

Figure 6: Plots showing orientations of all the interfaces for, (a) $D_{A A}=D_{B B}=2.0$, and (b) $D_{A A}=2.0, D_{B B}=1.0$, during three phase eutectic growth in a model symmetric ternary alloy. A single wavelength of the eutectic solids has the configuration $\alpha \beta \gamma$.

Figure 5: (Color online) Plots showing variations of $(\mathrm{d}) \alpha,(\mathrm{e}) \beta$, and (f) $\gamma$ phase compositions with $\lambda$, during three phase eutectic growth in a model symmetric ternary alloy. A single wavelength of the eutectic solids has the configuration: $\alpha \beta \gamma$. The figure legends can be interpreted in the same way as described in the caption of Fig. 1 
three-phase growth [41, 42, rather is a growth mode that is selected, which has also been found in previous three-phase growth simulations although due to different conditions [7]. Clearly, this is a prediction that is impossible to derive from the theoretical calculations that we present in this paper, and is certainly a limitation of the applicability of such calculations. More elaborately, in order for the theoretical predictions to be effective, one must have the information about the steady-state growth mode that is either derived experimentally, or through simulations.

One of the reasons for the $\alpha \beta \gamma$ configurations to tilt is that there are no symmetry planes once the volume fractions of the phases become unequal. The next arrangement $\alpha \beta \alpha \gamma$ however, possesses, two mirror axes, passing through the $\beta$ and the $\gamma$ phases. Going by the symmetry arguments placed in a previous paper [19], a steadystate growth mode where the lamellae are aligned with the growth direction, is therefore expected. We repeat the simulation and analytical calculations for this configuration for different diffusivities, and the results are reported in Figs 7. It is important to note that for this configuration a short wavelength instability exists which results in the transformation of the $\alpha \beta \alpha \gamma$ to $\alpha \beta \gamma$ occurring below a critical wavelength (see discussion in [19]). Thereby, we limit our analysis to only the stable lamellar states. For these spacings, we again derive an excellent agreement for the undercooling vs spacing variations, volume fractions and the phase compositions, between phase-field simulations and theoretical predictions. Due to the variation in the stability regimes we have limited our calculations for the case of only unequal diffusivities $D_{A A}=1.0, D_{B B}=2.0$, as the stability region for the contrary case of $D_{A A}=2.0, D_{B B}=1.0$ is very small.

\section{Conclusions}

In this study, we derive an analytical theory to determine the interfacial undercoolings, volume fractions and compositions of the solid phases in directionally solidifying

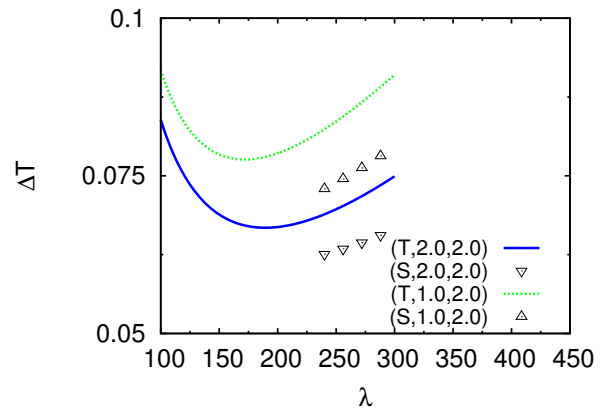

(a)

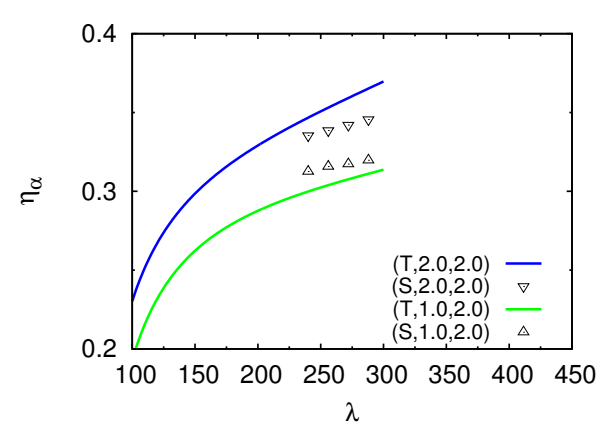

(b)

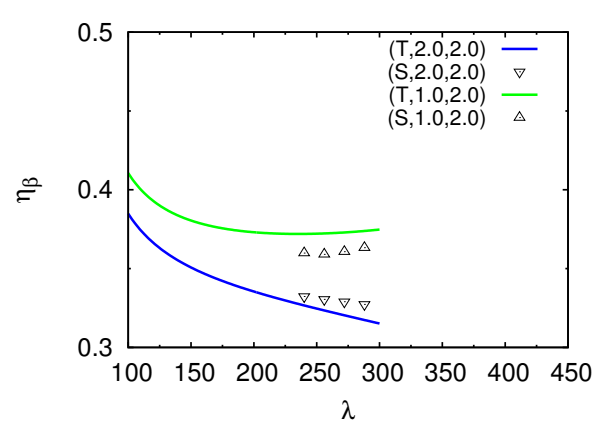

(c)

Figure 7: (Color online) Plots showing variations of (a) $\Delta T$, (b) $\eta_{\alpha}$, and (c) $\eta_{\beta}$, with $\lambda$, during three phase eutectic growth in a model symmetric ternary alloy. A single wavelength of the eutectic solids has the configuration $\alpha \beta \alpha \gamma$. The figure legends can be interpreted in the same way as described in the caption of Fig. 1 


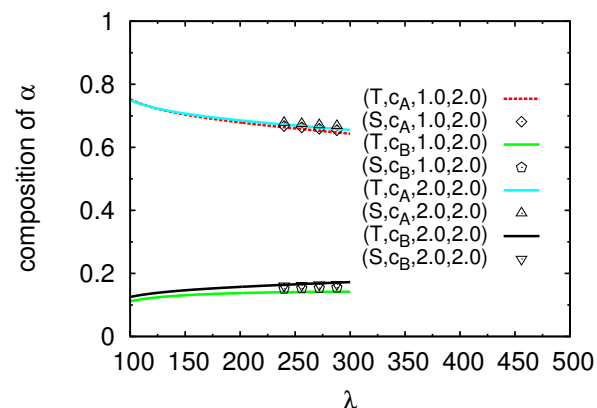

(d)

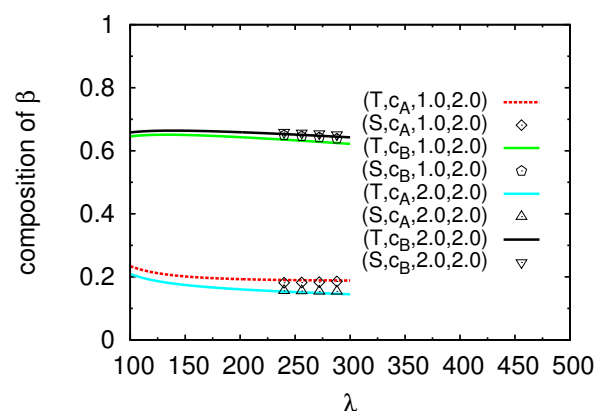

(e)

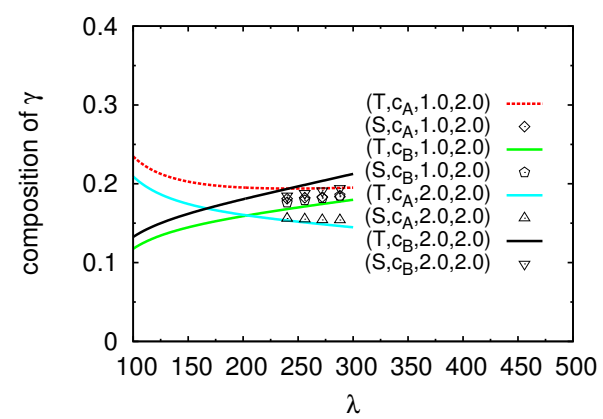

(f)

Figure 7: (Color online) Plots showing variations of (d) $\alpha,(\mathrm{e}) \beta$, and (f) $\gamma$ phase compositions with $\lambda$, in three phase eutectic growth in a model symmetric ternary alloy. A single wavelength of the eutectic solids has the configuration $\alpha \beta \alpha \gamma$. The figure legends can be interpreted in the same way as described in the caption of Fig. 1 lamellar eutectics for a generic multi-component, multiphase alloy. While our work bears similarities to the recently published work of Senninger and Voorhees [37] which is particular for two-phase growth, our work gives a generic prescription for treating any given multi-phase, multi-component alloy and in this respect can be seen as an extension of the previous work in [19]. A principal point in our theoretical calculations is that we treat the multi-variant and invariant eutectic reactions alike, by expressing the boundary layer compositions as functions of the respective state variables, which for our derivation are the diffusion potentials, the phase fractions and the undercooling. This allows us to solve the system of equations self-consistently for the undercoolings, phase fractions and the phase compositions along with the boundary layer compositions irrespective of the degrees of freedom in the system. Our derivation, thus unifies the method of theoretical calculations of the Jackson-Hunt type for any given multi-variant/invariant eutectic growth.

We also perform phase-field simulations to corroborate our theoretical predictions and they are found to be in reasonably good match with each other where we investigate the case of monovariant two-phase and three-phase invariant growth. Both the phase-field and the analytical theory exhibit the same trends in the variation of interfacial undercooling, solid phase volume fractions and compositions with change in lamellar width. It is important to highlight that the numerical differences in the predictions obtained from the two techniques are attributed to the assumption of a flat interface in the analytical calculations. Particularly, asymmetry in the interfacial shapes brought about either by strongly different phase fractions or interfacial energies result in asymmetric discrepancies between the theoretical predictions and the phase-field predictions. Thus we expect the match between the two methods w.r.t the predictions of the phase fractions and phase compositions to be the best for situations where the interfacial shapes of the phases are similar. Furthermore, we note in 
passing that while the theoretical expressions are generic in the spirit in which they have been derived, the existence of a steady-state lamellar growth mode needs to be ascertained through either phase-field simulations or experiments, before applying the results.

Secondly our study clearly highlights the importance of understanding the dependence of phase fractions on the diffusivity matrices. Changes in volume fractions can be associated with microstructural changes during two-phase growth(lamellar to rod), and many further possibilities during three-phase growth as seen in [43, 44]. Therefore, dependence of the volume fractions on the diffusivity matrices needs to be accounted for in order to derive a better understanding of pattern formation during bulk eutectic growth in multi-phase systems.

\section{Appendix}

The expression of the free energy of a solid phase $(p)$ is given by,

$$
f^{p}\left(\mathbf{c}^{\mathbf{p}}, T\right)=\sum_{i=1, j=1, i<=j}^{K-1, K-1} A_{i j}^{p} c_{i}^{p} c_{j}^{p}+\sum_{j=1}^{K-1} B_{j}^{p}(T) c_{j}^{p}+D^{p}(T),
$$

and that of the liquid is given by,

$$
f^{l}\left(\mathbf{c}^{\mathbf{l}}\right)=\sum_{i=1, j=1, i<=j}^{K-1, K-1} A_{i j}^{l} c_{i}^{l} c_{j}^{l}
$$

where the constants $A_{i j}^{p, l}$ are set to obtain $\left[\frac{\partial c^{p, l}}{\partial \mu}\right]$ matrices while $B_{j}^{p}$ and $D^{p}$ are determined from the equilibrium between solid and the liquid phases at a particular temperature as described in [45].

\section{References}

[1] H. Kerr, A. Plumtree, W. Winegard, Structure of tin-leadcadmium eutectic, Journal of the Institute of Metals 93 (2) (1964) 63.

[2] D. Cooksey, A. Hellawell, The microstructures of ternary eutectic alloys in the systems cd-sn-pb, in, tl-, al-cu-mg, zn, ag-, and zn-sn-pb, J INST METALS 95 (6) (1967) 183-187.
[3] H.-Q. Bao, F. Durand, Morphologie eutectique dans le système cd-pb-sn, Journal of Crystal Growth 15 (4) (1972) 291-295.

[4] M. Rinaldi, R. Sharp, M. Flemings, Growth of ternary composites from the melt: Part ii, Metallurgical Transactions 3 (12) (1972) 3139-3148.

[5] J. D. Holder, B. F. Oliver, The directional solidification of pbsn-cd alloys, Metallurgical Transactions 5 (11) (1974) 24232437.

[6] M. Ruggiero, J. Rutter, Origin of microstructure in $350 \mathrm{k}$ eutectic of bi-in-sn ternary system, Materials science and technology 11 (2) (1995) 136-142.

[7] U. Hecht, L. Gránásy, T. Pusztai, B. Böttger, M. Apel, V. Witusiewicz, L. Ratke, J. De Wilde, L. Froyen, D. Camel, et al., Multiphase solidification in multicomponent alloys, Materials Science and Engineering: R: Reports 46 (1) (2004) 1-49.

[8] S. Rex, B. Böttger, V. Witusiewicz, U. Hecht, Transient eutectic solidification in in-bi-sn: two-dimensional experiments and numerical simulation, Materials Science and Engineering: A 413 (2005) 249-254.

[9] V. Witusiewicz, L. Sturz, U. Hecht, S. Rex, Phase equilibria and eutectic growth in quaternary organic alloys amino-methyl-propanediol-(d) camphor-neopentylglycolsuccinonitrile (ampd-dc-npg-scn), Journal of crystal growth 297 (1) (2006) 117-132.

[10] R. Contieri, C. Rios, M. Zanotello, R. Caram, Growth and three-dimensional analysis of a nb-al-ni ternary eutectic, Materials Characterization 59 (6) (2008) 693-699.

[11] A. L. Genau, L. Ratke, Crystal orientation and morphology in al-ag-cu ternary eutectic, in: IOP Conference Series: Materials Science and Engineering, Vol. 27, IOP Publishing, 2012, p. 012032 .

[12] A. Dennstedt, L. Ratke, Microstructures of directionally solidified al-ag-cu ternary eutectics, Transactions of the Indian Institute of Metals 65 (6) (2012) 777-782.

[13] A. Dennstedt, L. Ratke, A. Choudhury, B. Nestler, New metallographic method for estimation of ordering and lattice parameter in ternary eutectic systems, Metallography, Microstructure, and Analysis 2 (3) (2013) 140-147.

[14] W. Brandt, Solution of the diffusion equation applicable to the edgewise growth of pearlite, Journal of Applied Physics 16 (3) (1945) 139-146.

[15] C. Zener, Kinetics of the decomposition of austenite, Trans. Aime 167 (550) (1946) e591.

[16] M. Hillert, The role of interfacial energy during solid state phase transformations, Jernkontorets Annaler 141 (1957) 757-789.

[17] K. Jackson, J. Hunt, Lamellar and rod eutectic growth, AIME Met Soc Trans 236 (1966) 1129-1142.

[18] T. Himemiya, T. Umeda, Three-phase planar eutectic growth 
models for a ternary eutectic system, Materials Transactions, JIM 40 (7) (1999) 665-674.

[19] A. Choudhury, M. Plapp, B. Nestler, Theoretical and numerical study of lamellar eutectic three-phase growth in ternary alloys, Phys. Rev. E 83 (2011) 051608.

[20] J. De Wilde, L. Froyen, S. Rex, Coupled two-phase $[\alpha($ al $)+\theta$ (al $2 \mathrm{cu}$ )] planar growth and destabilisation along the univariant eutectic reaction in al-cu-ag alloys, Scripta materialia 51 (6) (2004) 533-538.

[21] I. Yamauchi, S. Ueyama, I. Ohnaka, Effects of mn and co addition on morphology of unidirectionally solidified fesi2 eutectic alloys, Materials Science and Engineering: A 208 (1) (1996) 101 -107 .

[22] G. Garmong, The directional solidification of al- cu- mg monovariant alloys, Metallurgical and Materials Transactions B 2 (8) (1971) 2025-2030.

[23] P. Fehrenbach, H. Kerr, P. Niessen, Unidirectional solidification of monovariant eutectic cu-mg-ni alloys: I. planar interface stability criterion, Journal of Crystal Growth 16 (3) (1972) 209214.

[24] F. Schnake, G. Varchavsky, Microstructures of unidirectionally solidified al-rich cu-si alloys, Materials Characterization 39 (2-5) (1997) $345-359$.

[25] S. Raj, I. Locci, Microstructural characterization of a directionally-solidified ni-33 (at.\%) al-31cr-3mo eutectic alloy as a function of withdrawal rate, Intermetallics 9 (3) (2001) $217-227$.

[26] W. W. Mullins, R. Sekerka, Stability of a planar interface during solidification of a dilute binary alloy, Journal of applied physics 35 (2) (1964) 444-451.

[27] S. Akamatsu, G. Faivre, Traveling waves, two-phase fingers, and eutectic colonies in thin-sample directional solidification of a ternary eutectic alloy, Physical Review E 61 (4) (2000) 3757.

[28] S. Akamatsu, M. Perrut, S. Bottin-Rousseau, G. Faivre, Spiral two-phase dendrites, Physical review letters 104 (5) (2010) 056101 .

[29] M. Plapp, A. Karma, Eutectic colony formation: A stability analysis, Physical Review E 60 (6) (1999) 6865.

[30] M. Plapp, A. Karma, Eutectic colony formation: A phase-field study, Physical Review E 66 (6) (2002) 061608.

[31] D. G. Mccartney, J. D. Hunt, R. M. Jordan, The structures expected in a simple ternary eutectic system: Part 1. theory, Metallurgical Transactions A 11 (8) (1980) 1243-1249.

[32] L. Donaghey, W. Tiller, On the diffusion of solute during the eutectoid and eutectic transformations, part i, Materials Science and Engineering 3 (4) (1968) 231 - 239.

[33] A. Ludwig, S. Leibbrandt, Generalised 'jackson-hunt' model for eutectic solidification at low and large peclet numbers and any binary eutectic phase diagram, Materials Science and Engineering: A 375-377 (2004) $540-546$.

[34] P. Magnin, R. Trivedi, Eutectic growth: A modification of the jackson and hunt theory, Acta metallurgica et materialia 39 (4) (1991) 453-467.

[35] J. De Wilde, L. Froyen, V. T. Witusiewicz, U. Hecht, Two-phase planar and regular lamellar coupled growth along the univariant eutectic reaction in ternary alloys: An analytical approach and application to the al-cu-ag system, Journal of Applied Physics 97 (11).

[36] A. V. Catalina, P. W. Voorhees, R. K. Huff, A. L. Genau, A model for eutectic growth in multicomponent alloys, IOP Conference Series: Materials Science and Engineering 84 (1) (2015) 012085 .

[37] O. Senninger, P. W. Voorhees, Eutectic growth in two-phase multicomponent alloys, Acta Materialia 116 (2016) 308 - 320.

[38] A. Choudhury, B. Nestler, Grand-potential formulation for multicomponent phase transformations combined with thin-interface asymptotics of the double-obstacle potential, Phys.Rev.E 85 (2011) 021602.

[39] A. Choudhury, Quantitative phase-field model for phase transformations in multi-component alloys, Vol. Band 21, KIT Scientific Publishing, 2012.

[40] R. Folch, M. Plapp, Quantitative phase-field modeling of twophase growth, Phys. Rev. E 72 (2005) 011602.

[41] A. Karma, A. Sarkissian, Morphological instabilities of lamellar eutectics, Metallurgical and Materials Transactions A 27 (3) (1996) 635-656.

[42] S. Bottin-Rousseau, M. Şerefoğlu, S. Yücetürk, G. Faivre, S. Akamatsu, Stability of three-phase ternary-eutectic growth patterns in thin sample, Acta Materialia 109 (2016) 259-266.

[43] A. Choudhury, Y. C. Yabansu, S. R. Kalidindi, A. Dennstedt, Quantification and classification of microstructures in ternary eutectic alloys using 2-point spatial correlations and principal component analyses, Acta Materialia 110 (2016) 131-141.

[44] A. Lahiri, A. Choudhury, Effect of surface energy anisotropy on the stability of growth fronts in multiphase alloys, Transactions of the Indian Institute of Metals 68 (6) (2015) 1053-1057.

[45] A. Choudhury, Pattern-formation during self-organization in three-phase eutectic solidification, Transactions of the Indian Institute of Metals 68 (6) (2015) 1137-1143. 